\title{
Imperceptible-Visible Watermarking to Information Security Tasks in Color Imaging
}

\author{
Oswaldo Ulises Juarez-Sandoval ${ }^{1, *} \mathbb{B}$, Francisco Javier Garcia-Ugalde ${ }^{1}\left(\mathbb{D}\right.$, Manuel Cedillo-Hernandez $^{2}$, \\ Jazmin Ramirez-Hernandez ${ }^{2}$ and Leobardo Hernandez-Gonzalez ${ }^{2, *}$ (D) \\ 1 Facultad de Ingeniería, Universidad Nacional Autónoma de México (UNAM), Av. Universidad No. 3000, \\ Ciudad Universitaria, Coyoacán, México City 04510, Mexico; fgarciau@unam.mx \\ 2 Instituto Politécnico Nacional (IPN), Escuela Superior de Ingeniería Mecánica y Eléctrica, Unidad Culhuacan, \\ Av. Santa Ana No. 1000, San Francisco Culhuacan, Coyoacán, México City 04430, Mexico; \\ mcedilloh@ipn.mx (M.C.-H.); jramirezhe@ipn.mx (J.R.-H.) \\ * Correspondence: ojuarezs@ipn.mx (O.U.J.-S.); lhernandezg@ipn.mx (L.H.-G.)
}

Citation: Juarez-Sandoval, O.U.; Garcia-Ugalde, F.J.; CedilloHernandez, M.; Ramirez-Hernandez, J.; Hernandez-Gonzalez, L. Imperceptible-Visible Watermarking to Information Security Tasks in Color Imaging. Mathematics 2021, 9, 2374. https://doi.org/10.3390/math9192374

Academic Editors: Akemi

Galvez Tomida, Lihua You,

Hassan Ugail, Andres Iglesias Prieto and Alexander Malyshev

Received: 27 July 2021

Accepted: 17 September 2021

Published: 24 September 2021

Publisher's Note: MDPI stays neutral with regard to jurisdictional claims in published maps and institutional affiliations.

Copyright: (c) 2021 by the authors. Licensee MDPI, Basel, Switzerland. This article is an open access article distributed under the terms and conditions of the Creative Commons Attribution (CC BY) license (https:/ / creativecommons.org/licenses/by/ $4.0 /)$.

\begin{abstract}
Digital image watermarking algorithms have been designed for intellectual property, copyright protection, medical data management, and other related fields; furthermore, in real-world applications such as official documents, banknotes, etc., they are used to deliver additional information about the documents' authenticity. In this context, the imperceptible-visible watermarking (IVW) algorithm has been designed as a digital reproduction of the real-world watermarks. This paper presents a new improved IVW algorithm for copyright protection that can deliver additional information to the image content. The proposed algorithm is divided into two stages: in the embedding stage, a human visual system-based strategy is used to embed an owner logotype or a $2 \mathrm{D}$ quick response $(\mathrm{QR})$ code as a watermark into a color image, maintaining a high watermark imperceptibility and low image-quality degradation. In the exhibition, a new histogram binarization function approach is introduced to exhibit any watermark with enough quality to be recognized or decoded by any application, which is focused on reading QR codes. The experimental results show that the proposed algorithm can embed one or more watermark patterns, maintaining the high imperceptibility and visual quality of the embedded and the exhibited watermark. The performance evaluation shows that the method overcomes several drawbacks reported in previous algorithms, including geometric and image processing attacks such as JPEG and JPEG2000.
\end{abstract}

Keywords: invisible-visible watermarking; imperceptible-visible watermarking; unseen-visible watermarking; QR; histogram binarization function

\section{Introduction}

Since the beginning of the digital era and the rise of social media, many digital images and videos have been manipulated and shared without restrictions, control, and protection, creating copyright protection and intellectual propriety problems [1-4]. As a solution, several visible and invisible digital watermarking algorithms have been proposed mainly for images [5-7], video, audio [8,9], and other related fields [10-12]. However, in realworld applications such as banknotes, official documents, etc., the watermarks are used to deliver information about its authenticity. In this way, a novel watermarking algorithm named invisible-visible watermarking has been proposed as digital representation of the watermarks in real-world applications; in this algorithm, the imperceptibility, payload, and robustness of the invisible and the visible watermarking algorithms are combined with the high quality, resolution [13,14], and the robust display processing capabilities [14-17] incorporated into several electronic devices. This new watermarking technology can be classified by its exhibition stage as unseen-visible watermarking (UVW) [18-21] and imperceptible-visible watermarking (IVW) [22-25], as shown in Figure 1. 


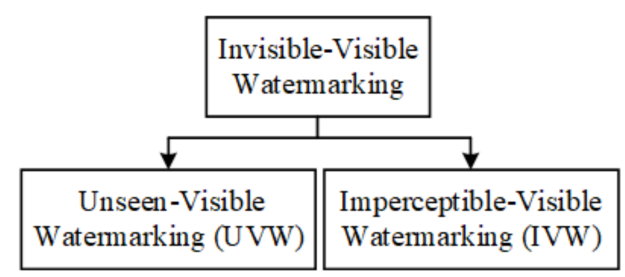

Figure 1. Invisible-visible watermarking classification.

The UVW algorithms [18-21] embed imperceptibly a watermark pattern through a visible watermarking strategy and take advantage of the image enhancement functions provided by the display devices, such as brightness, contrast, gamma, etc. [26,27], to exhibit without additional computational algorithms the watermark to the human visual system (HVS). The IVW algorithms [22-25] have been proposed as a solution to the several drawbacks presented in the exhibition stage of the UVW algorithms, where a QR code as a watermark is exhibited to the HVS in gray-color, which reduces its readability and the capacity to be correct decoded by common applications. Considering the mentioned drawbacks, the authors of [23] present a pioneer IVW algorithm that takes advantage of the processing capabilities incorporated into the capture devices to embed and exhibit a $\mathrm{QR}$ code as a watermark. This algorithm addresses and improves the drawbacks presented by the UVW algorithms; however, its applicability is restricted to professional capture devices with high storage memory and histogram modulation functions, and the exhibited watermark is shown to the HVS in color, which limits its reading and the information decoding by applications with the ability to read QR codes in color. Conversely, an improved IVW algorithm is presented in [24] that solves the above-mentioned drawbacks. To achieve this, a visible watermarking algorithm in the spatial domain is used to embed a QR code into a color image, and an invisible watermarking algorithm in the frequency domain [28] is used to embed the crucial parameters of the embedding stage. In the exhibition stage, the algorithm takes advantage of the processing capabilities of the electronics devices to exhibit the watermark pattern through a strategy named binarization function.

In this paper, the algorithm reported in [23] is improved to deliver additional information about the visual content of images via QR codes exhibited in black-and-white color, maintaining its capability to be exhibited in color; besides, it can be applied to information security tasks that do not require strong robustness. The proposed improvements consider in the embedding stage the human visual perception based on the just noticeable distortion (JND) [29] and the analysis of the histogram distortion induced by the embedding strategy [25]. In the exhibition stage, the algorithm takes advantages of the processing capabilities of the electronic devices to implement a histogram binarization function and exhibit the watermark in black-and-white color. The experimental results show that the proposed algorithm is capable of embedding a watermark maintaining a high imperceptibility and improving the performance of the previously reported algorithms [23,24], and its robustness to common geometric and image processing attacks is higher in several aspects compared with article [24], including JEPG and JPEG2000 compression.

The rest of this paper is organized as follows. Section 2 shows the related works of the IVW algorithms. The procedure of the proposed algorithm is presented in Section 3. The experimental results and a performance comparison with the algorithms [23] and [24] are shown in Sections 4 and 5, respectively. A discussion is presented in Section 6. Finally, this work concludes in Section 7.

\section{Related Works}

In this section, the IVW algorithms [23] and [24] are described briefly. These algorithms are considered fundamental works and are used to compare the performance of the proposed algorithm. 


\subsection{Imperceptible Visible Watermarking (IVW)}

The authors of [23] reported a pioneer IVW algorithm that is based on the observation of how several capture devices are equipped with robust image processing capabilities used to provide post-capture functions such as histogram modulation, which is considered as the exhibition strategy, as shown in Figure 2.

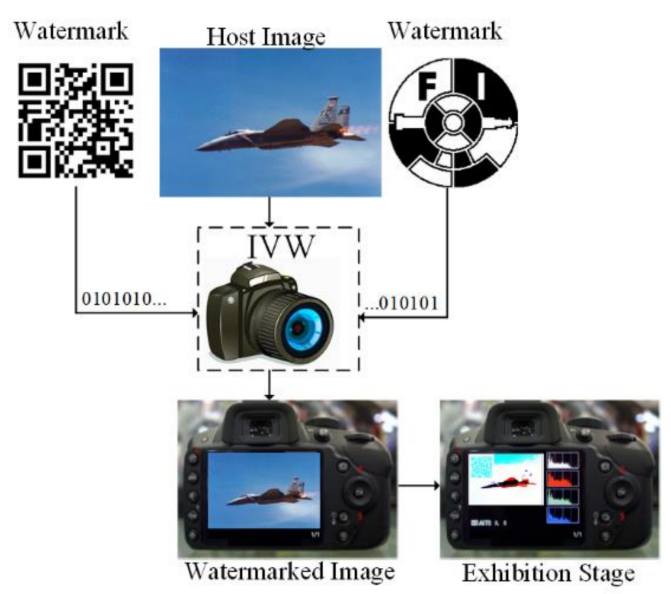

Figure 2. General diagram of the IVW.

In the algorithm, the appropriate embedding region is selected as the largest homogeneous region with smallest variance $R_{k}$ and size $M \times N$, from the color image $I_{k}$, where $k \in\{R, G, B\}$. Here, the appropriate mean value $m$ is satisfied by Equation (1).

$$
m= \begin{cases}\min \left(\bar{R}_{R}, \bar{R}_{G}, \bar{R}_{B}\right), & \text { if } \min \left(\bar{R}_{R}, \bar{R}_{G}, \bar{R}_{B}\right) \geq T, \\ \max \left(\bar{R}_{R}, \bar{R}_{G}, \bar{R}_{B}\right), & \text { otherwise, }\end{cases}
$$

where $\bar{R}_{k}$ and $T=2$ correspond to the mean value of the embedding region in the color channel $k$ and an empirical strength of the embedding strategy, respectively. To embed a binary watermark pattern $W=\left\{w_{i} \mid w_{i}=0,1\right.$, and $\left.i=1,2,3, \ldots, M \times N\right\}$, a color channel of the embedding region is selected among $R_{R}, R_{G}$, and $R_{B}$ with the smallest mean value. Finally, the embedding strategy is satisfied by Equation (2) if $\min \left(\bar{R}_{R}, \bar{R}_{G}, \bar{R}_{B}\right) \geq T$ or by Equation (3) if $\min \left(\bar{R}_{R}, \bar{R}_{G}, \bar{R}_{B}\right)<T$, respectively.

$$
\begin{aligned}
& R_{w}\left(p_{i}\right)= \begin{cases}m, & \text { if } w_{i}=0 \text { and } R_{k}\left(p_{i}\right)<m, \\
m-T, & \text { if } w_{i}=1 \text { and } R_{k}\left(p_{i}\right)>m-T,\end{cases} \\
& R_{w}\left(p_{i}\right)= \begin{cases}m+T, & \text { if } w_{i}=0 \text { and } R_{k}\left(p_{i}\right)<m+T, \\
m, & \text { if } w_{i}=1 \text { and } R_{k}\left(p_{i}\right)>m,\end{cases}
\end{aligned}
$$

where $R_{k}\left(p_{i}\right)$ and $R_{w}\left(p_{i}\right)$ are the $i$-th pixel value of the embedding and its corresponding watermarked region in the color channel $k$. Once the watermarked image $I_{w}$ is obtained, to exhibit the watermark, the algorithm leverages the processing capabilities equipped into the capture devices to execute an exhibition strategy based on the histogram modulation function in the range $[\alpha, \beta]$, where the range value is obtained as shown in Equation (4).

$$
\left\{\begin{array}{l}
\alpha=m-T, \beta=m, \quad \text { if } \min \left(\bar{R}_{R}, \bar{R}_{G}, \bar{R}_{B}\right) \geq T, \\
\alpha=m, \quad \beta=m+T, \quad \text { otherwise. }
\end{array}\right.
$$

\subsection{Improved Imperceptible Visible Watermarking (IIVW)}

The authors of [24] proposed an improved version of the IVW algorithm [23] that uses a visible watermarking algorithm in the spatial domain and an invisible water-marking algorithm based on the discrete cosine transform (DCT) [28] to embed imperceptibly a binary watermark into a color image $I_{k}$. As is shown in Figure 3, once the watermarked 
image is obtained, it is distributed to the end-users by on-demand or streaming services, and the exhibition stage leverages the processing capabilities of the display devices to exhibit the watermark to the HVS by the mechanism called binarization function.

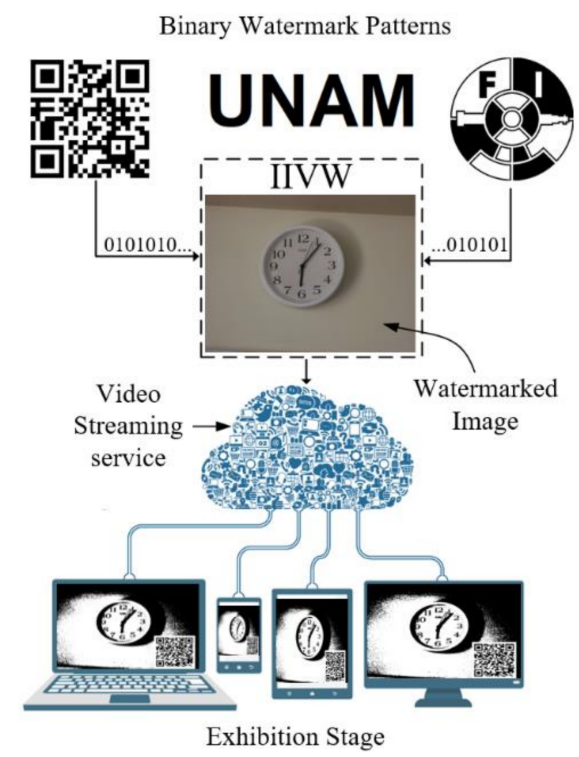

Figure 3. General diagram of the IIVW.

In this algorithm, $n$ binary watermarks $W=\left\{w_{i} \mid w_{i}=0,1\right.$, and $\left.i=1,2,3, \ldots, M \times N\right\}$ are embedded into $n$ non-overlapping embedding regions $R_{n} \in I_{k}$ selected with the smallest variance from the color image $I_{k}, k \in\{R, G, B\}$, as shown in Equation (5).

$$
R_{w}\left(p_{i}\right)= \begin{cases}\max \left(0, \mu_{R_{n}}-\Delta J N D\left(p_{i}\right)\right), & \text { if } w_{i}=0 \\ \min \left(\mu_{R_{n}}+\Delta J N D\left(p_{i}\right), 255\right), & \text { if } w_{i}=1\end{cases}
$$

where $\mu_{R n}$ and $R_{w}\left(p_{i}\right)$ are the mean value of the $n$-th region and the $i$-th pixel value of the watermarked region, respectively; $\Delta J N D\left(p_{i}\right)$ is the JND-based embedding strength [29], where $\Delta=0.5$ corresponds to the imperceptibility factor. After this process, a binary expression of the crucial parameters $\left\{n,\left(\mu_{R 1}, k_{1}\right),\left(\mu_{R 2}, k_{2}\right),\left(\mu_{R 3}, k_{3}\right), \ldots,\left(\mu_{R n}, k_{n}\right)\right\}$ is obtained to be embedded into BN blocks of size $(8 \times 8)$ through the imperceptible DCTbased watermarking algorithm called Quantization Index Modulation Dither-Modulation (QIM-DM) [28]. Finally, to exhibit the watermarks to the HVS, the QIM-DM inverse process is used to recover the crucial parameters used by the mechanism called the binarization function that is given by Equation (6).

$$
I_{E}=\left\{\begin{array}{l}
1, \quad \text { if } I_{k} \geq \mu_{R} \\
0, \text { otherwise, }
\end{array}\right.
$$

where $I_{E}$ corresponds to the exhibited image.

\subsection{Oportunities to Improve the IVW algorithms}

In the algorithm [23], the performance evaluation is reported in terms of the PSNR in the range of 49 to $69 \mathrm{~dB}$ for watermarks with sizes of $320 \times 320$ and $48 \times 48$, respectively. However, its embedding strategy, which is given by Equation (2) or Equation (3), modifies the histogram of the embedding region in the range of $[m-T, m]$ or $[m, m+T]$, as is shown in Figure $4 a$, b respectively. 


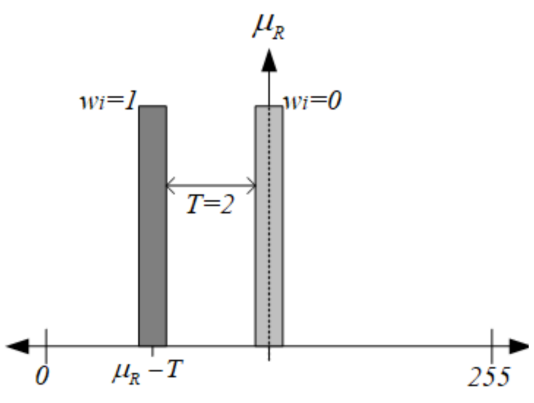

(a)

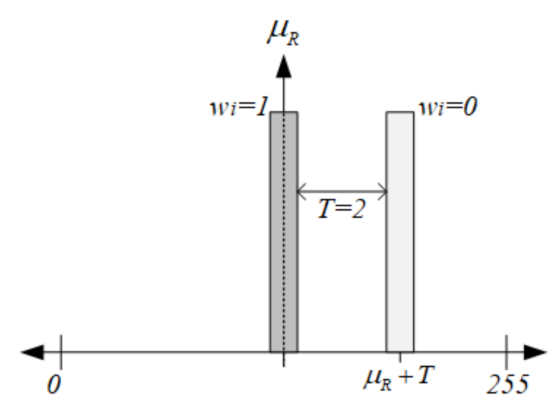

(b)

Figure 4. Histogram of the watermarked region. (a) Histogram of the watermarked region by Equation (2), (b) histogram of the watermarked region by Equation (3).

In the improved IVW algorithm [24], the performance evaluation is reported in terms of the PSNR in the range of 47.97 to $49.5 \mathrm{~dB}$ until three binary watermarks are embedded at the same time into a color image. However, in this algorithm, the embedding strategy given by Equation (5) modifies the histogram of the embedding region, as shown in Figure 5.

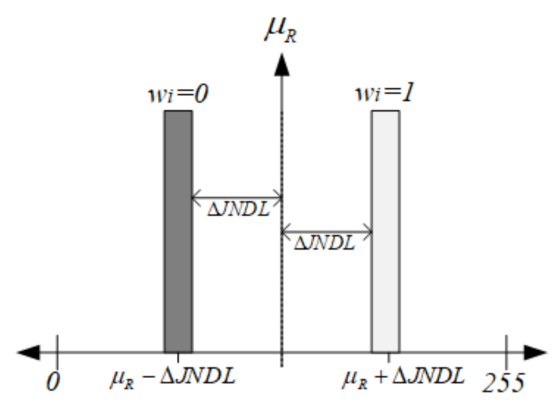

Figure 5. Histogram of the watermarked region by Equation (5).

As is described in Section 1, the algorithm in [24] improves the algorithm in [23] mainly in the exhibition stage where a QR code as watermark is exhibited in color, limiting its capability to be read. However, both algorithms have a strong similarity in their embedding strategies, which can be analyzed through the histogram of their corresponding watermarked regions. Comparing Figures 4 and 5, both algorithms accumulate the watermark information in the defined upper and lower boundaries with respect to the mean value; in the case of [23], there are $[m-T, m]$ and $[m, m+T]$ to 4 a,b respectively, and $\left[\mu_{R n}-\Delta J N D\left(p_{i}\right), \mu_{R n}+\Delta J N D\left(p_{i}\right)\right]$ to the improved IVW [24]; however, in [23], the watermark is exhibited in color to the HVS, representing an applicability limitation with respect to [24], where the watermark is exhibited in a black and white color that is a better option for QR codes as a watermark. Considering these similarities in the embedding stage, the histogram study of the IVW algorithms reported in [23,24], and observing Figures 4 and 5, in this paper, a new improved version of the IVW algorithms is proposed, where several weaknesses of the algorithm in [23] are improved by the performance of the algorithm in [24]. The embedding strategy is improved, substituting the empirical embedding strength $T \geq 2$ by the luminance-based just noticeable difference (JNDL), ensuring a high watermark imperceptibility under normal viewing condition. A new exhibition strategy called histogram binarization function is proposed as well, which exhibits the watermark pattern and binarizes the histogram of the watermarked image with respect to the mean value of the embedding region.

\section{Proposed Framework}

In the new improved IVW algorithm, a watermark pattern with several characteristics such as owner logotype or QR code can be embedded to provide in color copyright 
protection or deliver information about the image content; the procedure is divided into the embedding and exhibition stages. The embedding stage based on [23] is improved by the similarities of the algorithms reported previously [25], the histogram study of the invisible-visible watermarking algorithms [18-25], and the incorporation of the JNDL as embedding strength [29], as shown in Figure 6. The exhibition stage leverages the processing capabilities of the display devices to execute an exhibition strategy called histogram binarization function. In this section, both stages are described in detail.

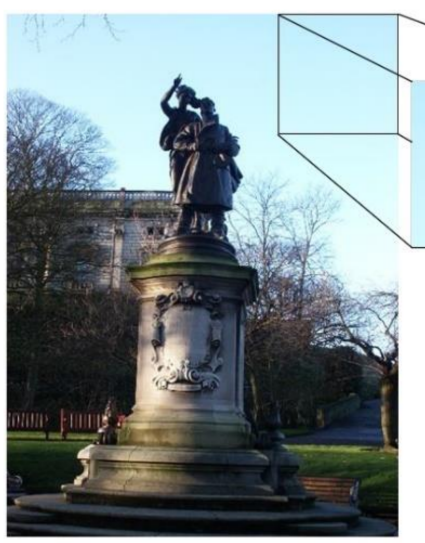

Original Image

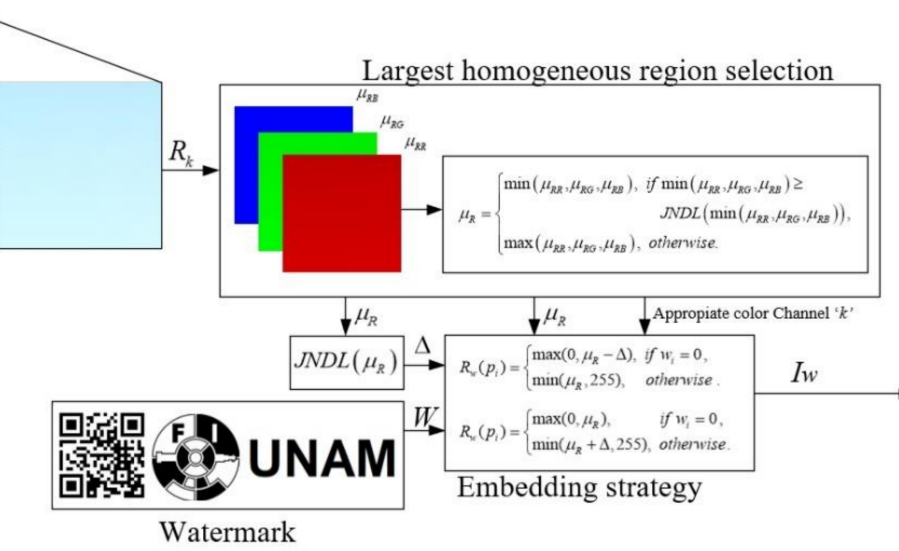

Watermark

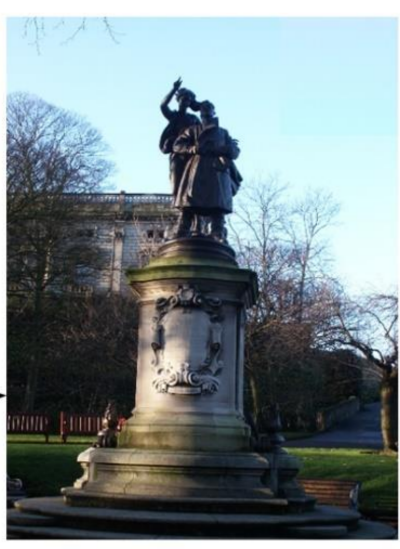

Watermarked Image

Figure 6. General diagram of the embedding stage.

\subsection{Embedding Stage}

Given an original color image $I_{k}=\left\{I_{k}\left(p_{i}\right) \mid 0 \leq I_{k}\left(p_{i}\right) \leq 255, i=1,2,3, \ldots, w_{I} \times h_{I}\right\}$, where $I_{k}\left(p_{i}\right)$ corresponds to the $i$-th pixel in the color channel $k \in\{R, G, B\}, w_{I}$ and $h_{I}$ are the width and height of the image, and a binary watermark pattern $W=\left\{w_{i} \mid w_{i}=0,1\right.$, and $i=1,2,3, \ldots, M \times N\}$, with size $M \times N$, the embedding procedure is given by the following steps.

1. Largest homogeneous region selection. In the beginning of the embedding procedure, an embedding region $R$, with the same size of the watermark, is selected as the largest homogeneous region from $I_{k}$; in other words, it must satisfy the minimum variance given by Equation (7).

$$
\min _{R \in I_{k}}\left(\frac{1}{(M \times N)-1} \sum_{i=1}^{M \times N}\left|R\left(p_{i}\right)-\mu *\right|\right)^{2},
$$

where $R\left(p_{i}\right)$ and $\mu^{*}$ are the $i$-th pixel of the largest homogeneous region and its corresponding mean value.

To ensure the homogeneity of the embedding region, a high percentage of its pixels must have the same intensity value; however, if the percentage of pixels is less than a predefined homogeneity threshold Th as shown in Equations (8) and (9), a smoothing operation can be used iteratively until minimize the variance among them.

$$
\sum_{i=1}^{M \times N} G\left(R\left(p_{i}\right)-\mu *\right) \leq M \times N \times T h
$$

where

$$
G(l)=\left\{\begin{array}{l}
1, \text { if } l=0 \\
0, \text { otherwise. }
\end{array}\right.
$$

Occasionally, the smoothing operation generates a strong visual degradation in the embedding region, which is perceived by the HVS under normal view conditions. To 
minimalize the visual degradation, instead of using a smoothing operation, the pixels with the most dispersed values are selected, and its values are replaced by others nearest to the mean value $\mu^{*}$; this strategy minimizes the variance and homogenizes the embedding region; empirical analysis shows that $10 \%$ of the pixels in $R$ can be used without affecting the visual content.

2. Appropriate color channel selection. Once the largest homogeneous region $R$ is obtained, an appropriate color channel is selected to embed the corresponding watermark $W$. According to the previously mentioned, the mean values of each color channel of the embedding region in an $R G B$ color model $\left(\mu_{R R}, \mu_{R G}\right.$, and $\left.\mu_{R B}\right)$ are obtained by Equation (10), and the appropriate color channel must satisfy Equation (11); for example, if the optimal mean value is satisfied by $\mu_{R}=\mu_{R G}$, in which $\mu_{R G}$ is the minimum or maximum mean value, the appropriate color channel corresponds to the green one.

$$
\left\{\begin{array}{l}
\mu_{R R}=\frac{1}{M \times N} \sum_{i=1}^{M \times N} R_{R}\left(p_{i}\right), \\
\mu_{R G}=\frac{1}{M \times N} \sum_{i=1}^{M \times N} R_{G}\left(p_{i}\right), \\
\mu_{R B}=\frac{1}{M \times N} \sum_{i=1}^{M \times N} R_{B}\left(p_{i}\right),
\end{array}\right.
$$

$\mu_{R}=\left\{\begin{array}{l}\min \left(\mu_{R R}, \mu_{R G}, \mu_{R B}\right), \quad \text { if } \min \left(\mu_{R R}, \mu_{R G}, \mu_{R B}\right) \geq J N D L\left(\min \left(\mu_{R R}, \mu_{R G}, \mu_{R B}\right)\right), \\ \max \left(\mu_{R R}, \mu_{R G}, \mu_{R B}\right), \text { otherwise, }\end{array}\right.$

where JNDL $(x)$ corresponds to luminance-based JND $[29,30]$.

3. Embedding strategy. In the proposed algorithm, the empirical embedding strength $T$ is improved by the just noticeable difference (JND), which represents the maximum luminance variation perceived by the HVS [29,30]. In the literature, several JND algorithms in the spatial and frequency domain have been reported to estimate a numerical value $[30,31]$. Its use is typically defined by the application [31-34], which in the proposed algorithm concerns the image watermarking. An embedding strength that allows embedding imperceptibly a visible watermark pattern is required; in this way, the JNDL function [29] is considered to estimate the embedding strength and ensure the watermark imperceptibility of the new improved IVW algorithm. Thus, if the appropriate color channel to embed the watermark pattern depends on ensuring that the largest homogeneous region $R$ has a variance value nearest to 0 , the JNDL-based embedding strength can be obtained with respect to the corresponding mean value $\mu_{R}$, as shown in Equation (12).

$$
\operatorname{JNDL}\left(\mu_{R}\right)=\left\{\begin{array}{cc}
-\frac{1}{8} \mu_{R}+6, & \mu_{R} \in(0,32) \\
-\frac{1}{32} \mu_{R}+3, & \mu_{R} \in(33,64) \\
\frac{1}{96} \mu_{R}+\frac{1}{3}, & \mu_{R} \in(65,255)
\end{array} \quad, 0 \leq \mu_{R} \leq 255\right.
$$

Once the largest homogeneous regions and its appropriate color channel are selected, an embedding strategy based on the JNDL is adopted to embed imperceptibly the binary watermark $W$. The embedding strategy is given by two ways given by Equations (13) and (14), which are satisfied when $\mu_{R}=\min \left(\mu_{R R}, \mu_{R G}, \mu_{R B}\right)$ or $\mu_{R}=\max \left(\mu_{R R}, \mu_{R G}, \mu_{R B}\right)$, respectively.

$$
\begin{gathered}
R_{w}\left(p_{i}\right)= \begin{cases}\max \left(0, \mu_{R}-\Delta\right), & \text { if } w_{i}=0, \\
\min \left(\mu_{R}, 255\right), & \text { otherwise, }\end{cases} \\
R_{w}\left(p_{i}\right)= \begin{cases}\max \left(0, \mu_{R}\right), & \text { if } w_{i}=0, \\
\min \left(\mu_{R}+\Delta, 255\right), & \text { otherwise, }\end{cases}
\end{gathered}
$$

where $R_{w}\left(p_{i}\right)$ corresponds to the $i$-th pixel of the watermarked region and $\Delta=\operatorname{round}\left(J N D L\left(\mu_{R}\right)\right)$ represents the embedding strength based on the JNDL with respect to the optimal mean value 
$\mu_{R}$. According to the embedding strategy given by Equations (13) and (14), the histogram of the watermarked region is modified as shown in Figure 7, ensuring the same distortion as that of the algorithms reported previously [23,24]. In both embedding strategies, the bits $w_{i}=0$ and $w_{i}=1$ of the binary watermark are embedded imperceptibly into the pixels with low and high intensity respectively, and with respect to the optimal mean value $\mu_{R}$. In Equation (13), the pixels with low intensity are far from the optimal mean value in $-\Delta$, as is shown in Figure 7a; whereas in Equation (14), the pixels with high intensity are far from the mean value in $+\Delta$, as is shown in Figure $7 \mathrm{~b}$.

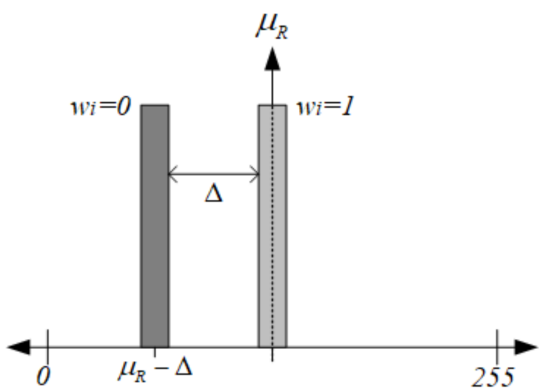

(a)

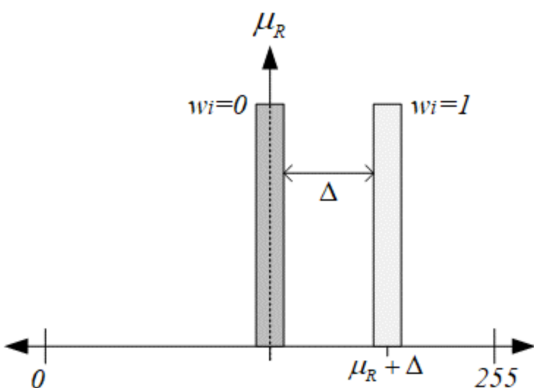

(b)

Figure 7. Histogram distortion of the watermarked region, (a) Histogram distortion induced by Equation (13), (b) Histogram distortion induced by Equation (14).

4. Finally, the watermarked image $I_{w}=\left\{I_{w}\left(p_{i}\right) \mid 0 \leq I_{w}\left(p_{i}\right) \leq 255, i=1,2,3, \ldots, w_{I w} \times\right.$ $\left.h_{I w}\right\}$ with size $w_{I w} \times h_{I w}$ is obtained through the reconstruction of the watermarked region $R_{w}$.

According to the embedding procedure survey in Table 1 and considering that $n$ non-overlapping homogeneous regions can be found into an image, these steps can be performed iteratively until they have embedded $n$ binary watermark patterns imperceptibly to the HVS. Although the proposed algorithm is designed to embed a watermark with its original size, it is not limited to leverage the robustness of the QR codes and the characteristics of owner logotypes to resize it if the original image does not have homogeneous regions with the same size as the watermark.

Table 1. Embedding procedure survey.

\begin{tabular}{|c|c|}
\hline Procedures & Details \\
\hline Input & Original image $I_{k}$ and binary watermark $W$ pattern \\
\hline Step 1 & $\begin{array}{l}\text { The largest homogeneous region } R \text { with the same size of the watermark pattern is selected from } \\
\text { the original color image. }\end{array}$ \\
\hline Step 2 & $\begin{array}{l}\text { The mean values } \mu_{R R}, \mu_{R G} \text {, and } \mu_{R B} \text { of each } R G B \text { color channel are obtained, as formulated by } \\
\text { Equation (10). }\end{array}$ \\
\hline Step 3 & $\begin{array}{l}\text { According to Equation (11), the optimal mean value } \mu_{R} \text { is obtained. Moreover, the minimum or } \\
\text { maximum mean value selected as the optimal one } \mu_{R} \text {, also determines the appropriate color } \\
\text { channel to embed the binary watermark pattern } W \text {. }\end{array}$ \\
\hline Step 4 & $\begin{array}{l}\text { Ensuring the homogeneity of the embedding region } R \text {, the embedding strength JNDL-based is } \\
\text { obtained with respect to the optimal mean value } \mu_{R} \text {, as shown in Equation (12). }\end{array}$ \\
\hline Step 5 & $\begin{array}{l}\text { According to Equation (13) or Equation (14), the binary watermark pattern } W \text { is embedded into } \\
\text { the appropriate embedding region. }\end{array}$ \\
\hline Step 6 & $\begin{array}{l}\text { Finally, once the watermark pattern } \mathrm{W} \text { is embedded, the watermarked region is reconstructed, } \\
\text { and the watermarked image Iw is obtained. }\end{array}$ \\
\hline Output & Watermarked image $I_{w}$ \\
\hline
\end{tabular}




\subsection{Exhibition Stage}

In the final stage of the proposed framework, the watermark is exhibited to the HVS by a novel histogram binarization function that leverages the characteristics of the embedding strategy and the histogram distortion induced with respect to the optimal mean value; also, this strategy can be easily implemented in several electronic devices.

\section{Histogram Binarization Function}

The two approaches of the embedding strategy given by Equations (13) and (14) modify the histogram of the appropriate color channel as shown in Figure 7, and its characteristics are leveraged to propose the novel histogram binarization function as the exhibition stage, as shown in Figure 8. The main idea of this function is to binarize the watermarked image $I_{w}$ with respect to the main histogram characteristic of the watermarked region, such as the optimal mean value $\mu_{R}$, through the two different functions formulated by Equations (15) and (16).

$$
\begin{aligned}
& I_{E}\left(p_{i}\right)= \begin{cases}1, & \text { if } \mu_{R}=\min \left(\mu_{R R}, \mu_{R G}, \mu_{R B}\right) \text { and } I_{w}\left(p_{i}\right) \geq \mu_{R}, \\
0, & \text { otherwise, }\end{cases} \\
& I_{E}\left(p_{i}\right)= \begin{cases}0, & \text { if } \mu_{R}=\max \left(\mu_{R R}, \mu_{R G}, \mu_{R B}\right) \text { and } I_{w}\left(p_{i}\right) \leq \mu_{R}, \\
1, & \text { otherwise, }\end{cases}
\end{aligned}
$$

where Equations (15) and (16) are satisfying the embedding strategy given by Equations (13) and (14), respectively. Finally, $I_{E}\left(p_{i}\right)$ corresponds to the $i$-th pixel of the exhibited image $I_{E}$.

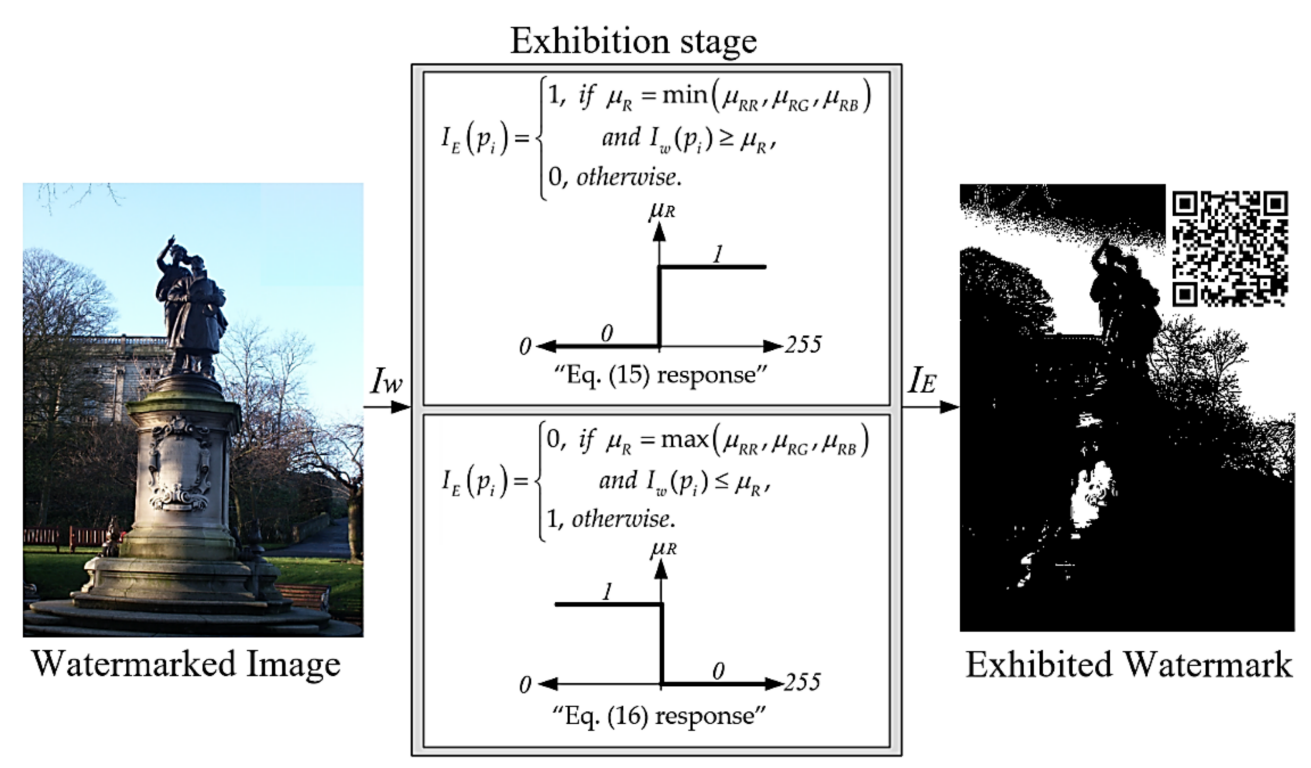

Figure 8. General diagram of the exhibition stage.

\section{Experimental Results}

According to [24], the robustness against malicious or non-malicious attacks becomes meaningless, since the main approach of the IVW algorithms is the additional information delivery to the image visual content. However, the copyright application of the proposed algorithm requires reconsidering a robustness evaluation [35]. The experimental results show their applicability to any class of images, and the performance evaluation is provided against common geometric and image processing attacks including JPEG and JPEG2000 compression. Additionally, this section includes a fair comparison against several previously reported algorithms. 


\subsection{Dataset Detail and Binary Watermark Pattern}

The dataset used to evaluate the performance of the proposed algorithm is obtained from the uncompressed color image dataset (UCID) [36], which contains 1338 uncompressed color images with $512 \times 384$ size and TIFF format. As shown in Figure 9, three binary watermark patterns are used for the performance evaluation, where Figure $9 a, c$ are two owner logotypes with sizes of $150 \times 150$ and $134 \times 39$, respectively. Figure $9 \mathrm{~b}$ corresponds to a $\mathrm{QR}$ code of $116 \times 116$ size and error correction label $\mathrm{L}$ that corresponds to a 7\% of loss information; additionally, to specifically evaluate QR codes, error correction levels $\mathrm{M}, \mathrm{Q}$, and $\mathrm{H}$ are capable of information decoding with $15 \%, 25 \%$, and $30 \%$ of loss information [37], respectively.

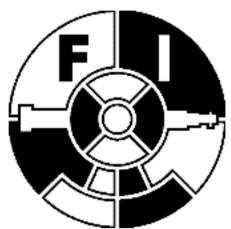

(a)

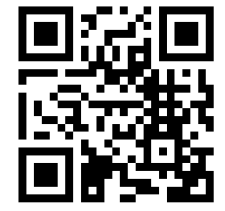

(b)

\section{UNAM}

(c)

Figure 9. Binary watermark patterns: (a) Owner logotype FI, (b) QR code level L, and (c) Owner logotype UNAM.

\subsection{Performance Metrics for Image Quality Degradation, Imperceptibility, and Robustness}

To evaluate the performance of the proposed algorithm in terms of the watermarked image quality degradation, imperceptibility of the watermark pattern, and robustness against the common geometric and image processing attacks listed in Tables 2 and 3, the peak signal to noise ratio (PSNR), structural similarity index (SSIM) [38], normalized color difference (NCD) [39], normalized correlation (NC), and bit error rate (BER) are used.

Table 2. Common geometric attacks used in the experiment.

\begin{tabular}{|c|c|}
\hline Attack & Specification \\
\hline Rotation & Angle $45^{\circ},\left\{90^{\circ}, 180^{\circ}, 270^{\circ}\right\}$ \\
\hline Rescaling & $0.75 \times,\{0.9 \times, 1.1 \times, 1.5 \times\}$ \\
\hline Translation with cropping & $x=50, y=50 ;\{x=-50, y=-50\}$ \\
\hline Flip transformation & Horizontal-\{Vertical $\}$ direction \\
\hline Affine Transformation & {$[1,0.3,0 ; 0.3,2,0 ; 0,0,1]$} \\
\hline Aspect ratio & {$[0.8,0,0 ; 0,1.5,0 ; 0,0,1]$} \\
\hline Shearing & 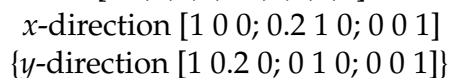 \\
\hline Radial & - \\
\hline
\end{tabular}

\{ \} additional experimental results refer to [40].

Image quality degradation is generally measured by the performance metric PSNR, as shown in Equation (17).

$$
\begin{gathered}
\operatorname{PSNR}\left(I_{k}, I_{w}\right)=10 \log _{10} \frac{\max \left(I_{k}\left(p_{i}\right)\right)^{2}}{M S E}, \\
\operatorname{MSE}\left(I_{k}, I_{w}\right)=\frac{1}{w_{I w} \times h_{I w}} \sum_{i=1}^{w_{I w} \times h_{I w}}\left[I_{k}\left(p_{i}\right)-I_{w}\left(p_{i}\right)\right]^{2},
\end{gathered}
$$

where MSE given by Equation (18) is the mean square error between the original $I_{k}$ and the watermarked image $I_{w}$, and $w_{I w}=w_{I}$ and $h_{I w}=h_{I}$ are the width and height of the watermarked image. Typically, when PSNR $\in(37 d B$, inf $)$, it means that the embedding process generates a low-quality degradation in the watermarked image, and a value nearest to $0 \mathrm{~dB}$ represents a higher-quality degradation [41]. 
Table 3. Common image processing attacks used for the experiment.

\begin{tabular}{|c|c|c|c|c|}
\hline \multirow{2}{*}{\multicolumn{2}{|c|}{ Attack }} & \multicolumn{3}{|c|}{ Specification } \\
\hline & & \multicolumn{3}{|c|}{ Image Watermarked by } \\
\hline & & FI & QR & UNAM \\
\hline \multirow{3}{*}{ Filter } & Average & $7 \times 7$ & $3 \times 3$ & $5 \times 5$ \\
\hline & Median & $5 \times 5$ & $3 \times 3$ & $9 \times 9$ \\
\hline & Gaussian & $9 \times 9$ & $3 \times 3$ & $9 \times 9$ \\
\hline \multirow{2}{*}{$\begin{array}{c}\text { Noise } \\
\text { contamination }\end{array}$} & Impulsive & density $=0.5$ & density $=0.3$ & density $=0.5$ \\
\hline & Gaussian & $\mu=0, \sigma^{2}=0.0004$ & $\begin{array}{c}\mu=0, \sigma^{2}= \\
0.00004\end{array}$ & $\mu=0, \sigma^{2}=0.0005$ \\
\hline Sharpening & & $\begin{array}{l}\text { Radius }=4, \\
\text { Amount }=1\end{array}$ & $\begin{array}{l}\text { Radius }=0.5 \\
\text { Amount }=2\end{array}$ & $\begin{array}{l}\text { Radius }=5, \\
\text { Amount }=2\end{array}$ \\
\hline \multirow{2}{*}{$\begin{array}{c}\text { Blurring } \\
\text { Histogram } \\
\text { Equalization }\end{array}$} & - & Radio $=4$ & Radio = 1.7 & Radio $=4$ \\
\hline & & {$[0,0.33]$} & {$[0,0.33]$} & {$[0,0.33]$} \\
\hline \multirow{2}{*}{ Compression } & JPEG & QF:90 & QF:90 & QF:75 \\
\hline & JPEG2000 & CR:10 & CR:10 & CR:15 \\
\hline
\end{tabular}

The imperceptibility can be defined as a measure of similarity between the original and its corresponding watermarked image. In addition to the PSNR, other metrics to measure the imperceptibility are the SSIM and the NCD, where the SSIM represents an individual comparison of three image parameters such as luminance, contrast, and structure, as shown in (19).

$$
\operatorname{SSIM}\left(I_{k}, I_{w}\right)=\frac{\left(2 \mu_{I_{k}} \mu_{I_{w}}+C_{1}\right)\left(2 \sigma_{I_{k}}, I_{w}+C_{2}\right)}{\left(\mu_{I_{k}}^{2}+\mu_{I_{w}}^{2}+C_{1}\right)\left(\sigma_{I_{k}}^{2} \sigma_{I_{w}}^{2}+C_{2}\right)},
$$

where $\mu_{I}$ and $\mu_{I w}$ are the mean value of $I_{k}$ and $I_{w w}$, respectively, $\sigma_{I, I w}$ and $\sigma^{2} X$ are the corresponding covariance and variance between $I_{k}$ and $I_{w}$, respectively, and $C_{1}=\left(k_{1} L\right)^{2}$ and $C_{2}=\left(k_{2} L\right)^{2}$ are two constants used to stabilize the denominator, where $k_{1}=0.01$, $k_{2}=0.03$ and $L=255$ correspond to the dynamic range of 8 bits per pixel. The NCD is another performance metric of imperceptibility that measures in the CIELAB color space the similarity between the original image $I_{k}$ and its corresponding watermarked image $I_{w}$; it is formulated as shown in (20).

$$
\operatorname{NCD}\left(I_{k}, I_{w}\right)=\frac{\sum_{x=1}^{h_{I w}} \sum_{y=1}^{w_{I}}\left(\sqrt{(\Delta L(x, y))^{2}+(\Delta a(x, y))^{2}+(\Delta b(x, y))^{2}}\right)}{\sum_{x=1}^{h_{I}} \sum_{y=1}^{w_{I}}\left(\sqrt{(L(x, y))^{2}+(a(x, y))^{2}+(b(x, y))^{2}}\right)},
$$

where $\Delta L(x, y), \Delta a(x, y)$, and $\Delta b(x, y)$ of the numerator are the difference between the luminance $L$ and the spaces $a$ and $b$ of the original image I and the watermarked image $I_{w}$ at the position $(x, y)$. In the denominator $L, a$ and $b$ are the original image color spaces. Generally, the SSIM and NCD values nearest to 1 and 0 , respectively, represent a strong similarity between the original image and its corresponding watermarked image.

The NC and the BER are two metrics used in this paper to evaluate the similarity between the original $W$ and the recover watermark patter $W^{\prime}$ as well as the robustness against the common geometric and imagen processing attacks listed in Tables 2 and 3, respectively. The NC and the BER are denoted by Equations (21) and (22).

$$
N C\left(W, W^{\prime}\right)=\frac{\sum_{i=1}^{M \times N}\left[w_{i} \times w_{i}^{\prime}\right]}{\sqrt{\sum_{i=1}^{M \times N}\left(w_{i}\right)^{2}} \times \sqrt{\sum_{i=1}^{M \times N}\left(w_{i}^{\prime}\right)^{2}}},
$$




$$
\operatorname{BER}\left(W, W^{\prime}\right)=\frac{\sum_{i=1}^{M \times N}\left(w_{i} \otimes w^{\prime}{ }_{i}\right)}{M \times N},
$$

where $\otimes, w_{i}$, and $w_{i}^{\prime}$ are the XOR function, $i$-th pixel of the original $W$, and the recover watermark $W^{\prime}=\left\{w_{i}^{\prime} \mid w^{\prime}{ }_{i}=0,1\right.$, and $\left.i=1,2,3, \ldots, M \times N\right\}$, with size $M \times N$, respectively. According to Equations (21) and (22), the values of NC and BER nearest to 1 and 0, respectively, represent a strong similarity and robustness against common geometric and imagen processing attacks.

Additionally, to evaluate the performance of the proposed algorithm with respect to the geometric and the image processing attacks listed in Tables 2 and 3, a fair performance comparison with the most sophisticated and recent IVW algorithms $[23,24]$ is provided.

\subsection{Suitability for Any Class of Images}

Similar to the most recent and sophisticated reported algorithms [24], the proposed algorithm can use any class of images to embed any binary watermarks, mainly QR codes. As mentioned above, to evaluate the performance of the proposed algorithm, the binary watermark patterns FI, QR, and UNAM are used.

Although not all images of the UCID dataset ensure the largest homogeneous region to embed the mentioned watermark patterns, the proposed algorithm is able to obtain a low visual quality degradation and high imperceptibility; e.g., Table 4 shows three images that ensure the largest homogeneous region with the minimum required variance and three that do not ensure it, and its corresponding exhibited watermark pattern. Additionally, its performance evaluation in terms of quality degradation, imperceptibility, and robustness is shown, wherein both kinds of images offer a good quantitative and visual performance in non-attacked images.

\subsection{Image Quality Degradation and Imperceptibility Analysis}

In an efficient watermarking algorithm, the image quality degradation is performed by the PSNR, which is strongly related with the imperceptibility that in terms of SSIM and NCD performs similarly between the original and its corresponding watermarked image. To obtain this evaluation, the binary watermark patterns FI, QR, and UNAM are embedded individually into the 1338 images contained within the UCID dataset, and the PSNR, SSIM, and NCD of the images with the same mean value $0 \leq \mu_{R} \leq 255$ in the embedding region are averaging. For the FI watermark, the average values of PSNR, SSIM, and NCD with respect to the mean value $\mu_{R}$ are shown in Figure 10a-c, respectively, those with respect to the $\mathrm{QR}$ watermark are shown in Figure 10d-f, respectively; and finally, those with respect to the UNAM watermark are shown in Figure $10 \mathrm{~g}-\mathrm{i}$, respectively.

Generally, if $P S N R \geq 37 \mathrm{~dB}$, the watermarked image quality is acceptable, and the watermark is considered invisible to the HVS [39]. In this way, Figure 10a,d,g show the average of the performance quality metric with a value greater than $37 \mathrm{~dB}$. Considering all the images of the mentioned dataset, the average of quality for each embedded watermark pattern FI, QR, and UNAM is greater than $37 \mathrm{~dB}, 43.50 \mathrm{~dB}$, and $53 \mathrm{~dB}$, respectively; in this context, the general quality value (GQV) of the proposed algorithm is $37 \mathrm{~dB} \leq G Q V \geq 53 \mathrm{~dB}$.

In the experiment, if $S S I M \geq 0.93$ and $N C D \leq 0.05$, the original image and its corresponding watermarking are considered similar, and the embedded watermark is considered as imperceptible to the HVS. Figure $10 \mathrm{~b}, \mathrm{c}, \mathrm{e}, \mathrm{f}, \mathrm{h}, \mathrm{i}$ show the imperceptibility metric SSIM and NCD with respect to the mean value $\mu_{R}$ of the appropriate embedding region. As can be observed, the obtained SSIM and NCD values are greater than 0.93 and smaller than 0.05 , respectively. Similar to the GQV, a general similarity value (GSV) and general imperceptibility value (GIV) for watermarks FI, QR, and UNAM are obtained. A survey of the general performance for the UCID dataset is shown in Table 5. 
Table 4. Visual quality degradation and imperceptibility of non-attacked watermarked images.

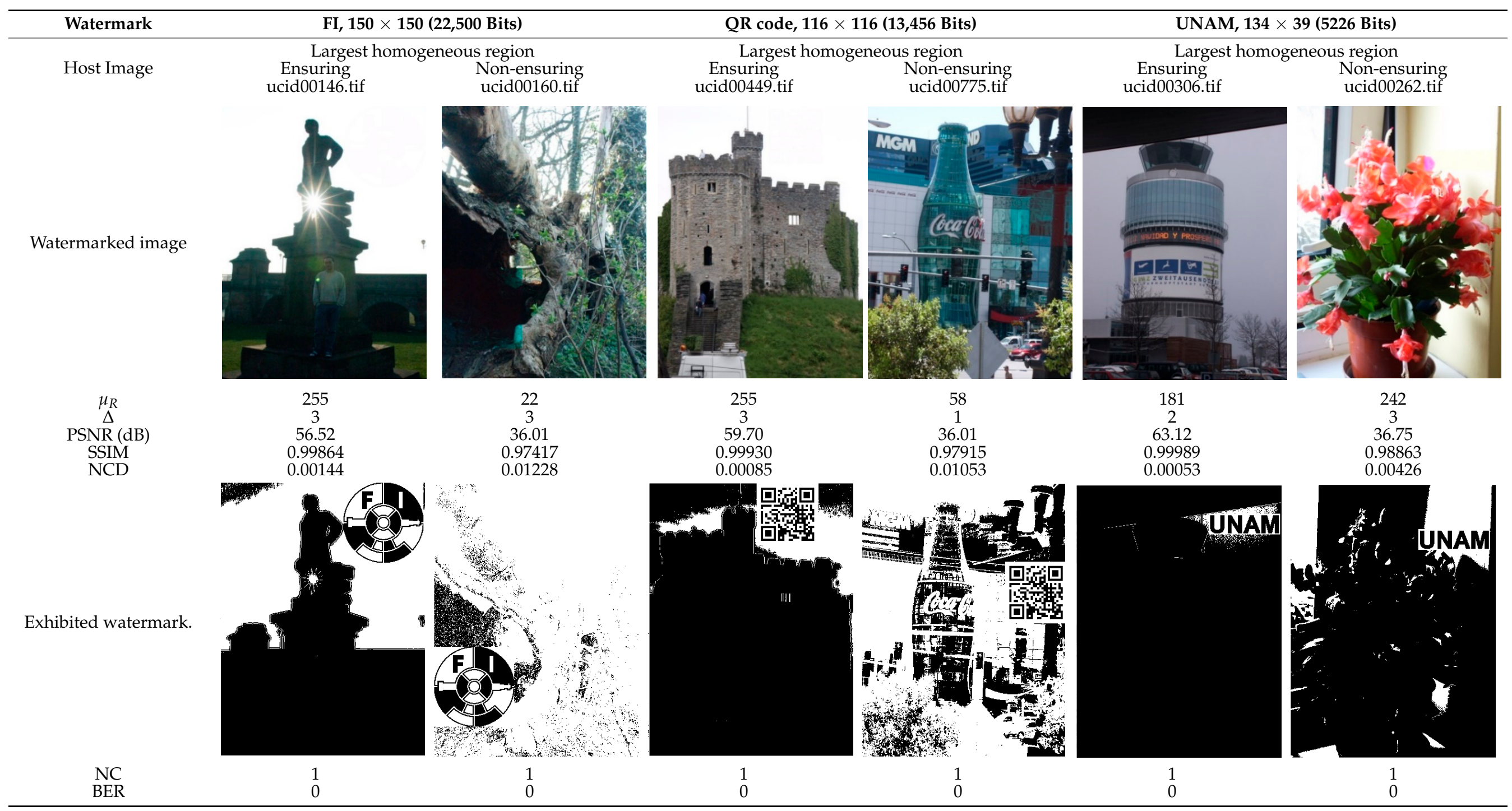




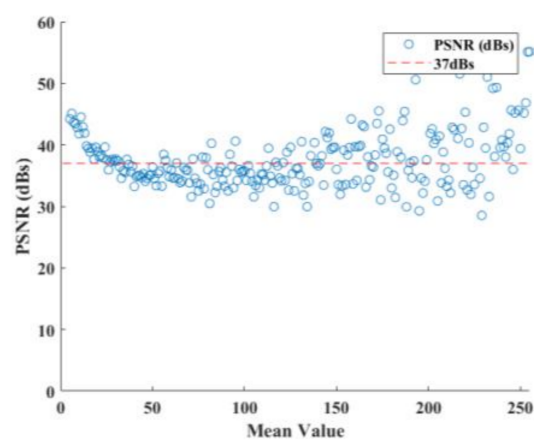

(a)

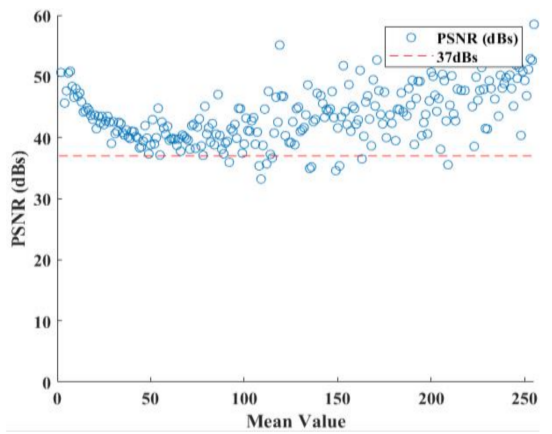

(d)

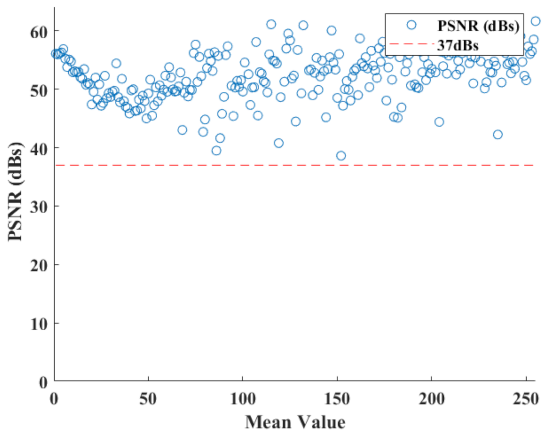

(g)

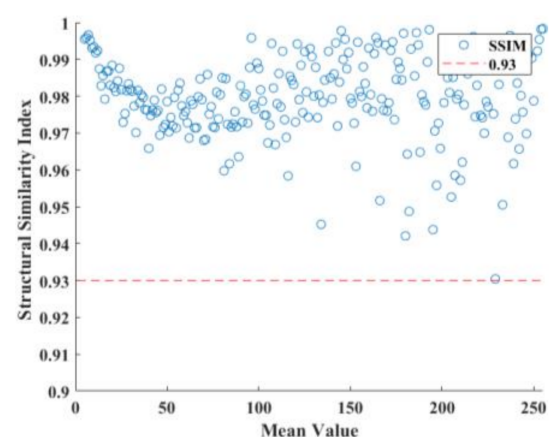

(b)

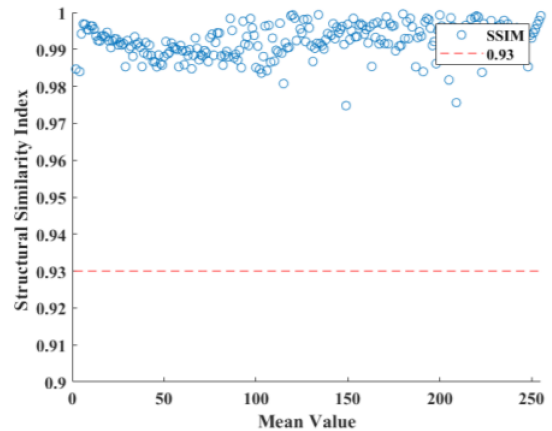

(e)

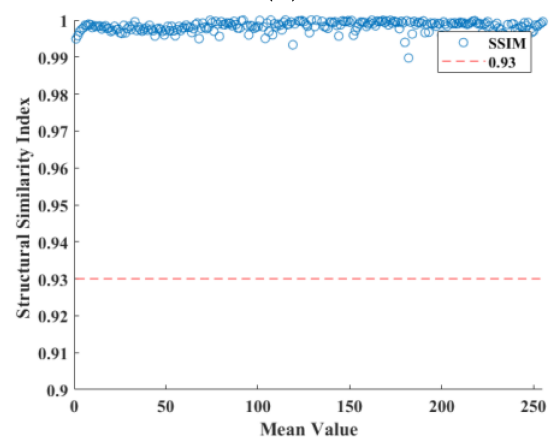

(h)

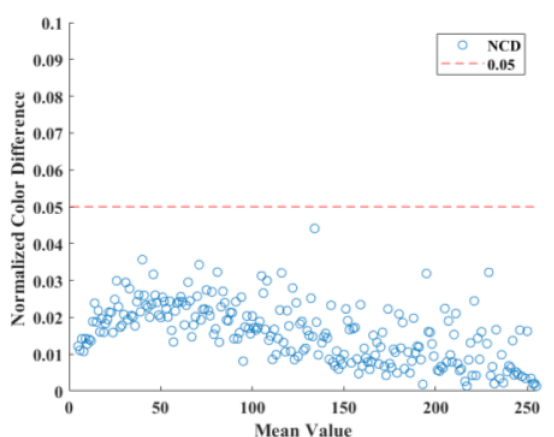

(c)

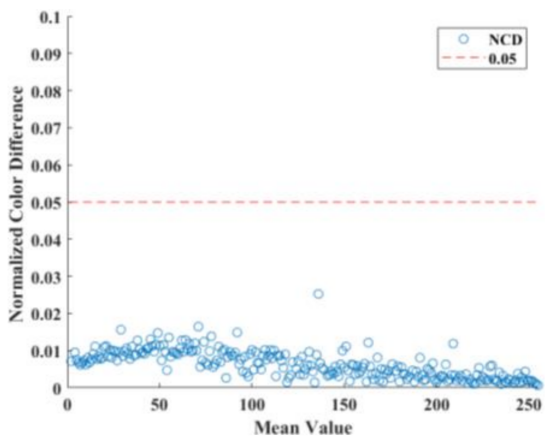

(f)

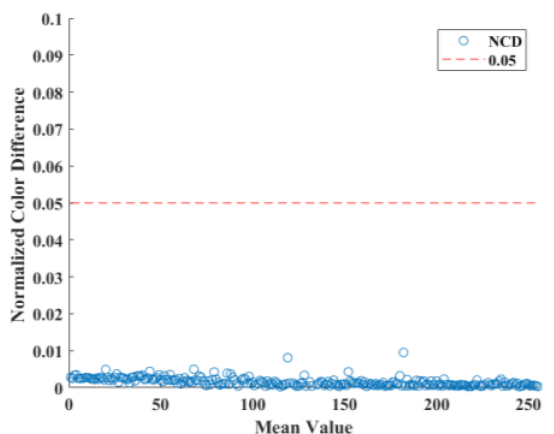

(i)

Figure 10. Watermarked UCID graphical quality degradation and imperceptibility. (a-c) are the corresponding PSNR, SSIM, and NCD for the FI watermark; (d-f) are the corresponding PSNR, SSIM, and NCD for the QR watermark; (g-i) are the corresponding PSNR, SSIM, and NCD for the UNAM watermark.

Table 5. General performance for UCID.

\begin{tabular}{cccccc}
\hline \multicolumn{2}{c}{ Image Size } & & & \\
Watermark & Length (Bits) & GQV (dB) & GSV & GIV \\
\cline { 1 - 3 } FI & 22,500 & & 37 & 0.9795 & 0.0188 \\
QR & 19,600 & 43.5 & 0.9920 & 0.0073 \\
UNAM & 5226 & 53 & 0.9982 & 0.0017 \\
\hline
\end{tabular}

\subsection{Robustness against Common Geometric Attacks}

The easy geometric edition of digital images by mobile applications [42] or dedicated software [43] requires the proposed algorithm performance evaluation against common geometric attacks [35], such as those listed in Table 2. In this paper, the QR codes are 
considered the most sensible watermark patterns, because additionally to the recognition, they also required the stored information decoding.

In this context, an image is watermarked by the QR watermark; Table 6 shows the robustness results of the attacked image and its corresponding exhibited watermark. However, due to paper length, several results regarding Rotation, Translation with cropping, Flip transformation, and Shearing attacks are omitted in Table 6 but can be accessed from [40], including similar experimental results to FI and UNAM watermarks.

Table 6. Robustness against geometric attacks.

\begin{tabular}{|c|c|c|c|c|}
\hline & Rotation $45^{\circ}$ & Rescaling $0.75 X$ & Affine Transformation & Aspect Ratio \\
\hline \multicolumn{5}{|l|}{$\begin{array}{l}\text { Attacked } \\
\text { image }\end{array}$} \\
\hline \multicolumn{5}{|l|}{$\begin{array}{c}\text { Exhibited } \\
\text { watermark }\end{array}$} \\
\hline \multirow[t]{2}{*}{ QR decoded } & Yes & Yes & No & No \\
\hline & $\begin{array}{l}\text { Translation with } \\
\text { cropping }\end{array}$ & Flip transformation & Shearing $x$-direction & Radial Transformation \\
\hline
\end{tabular}

Attacked
image

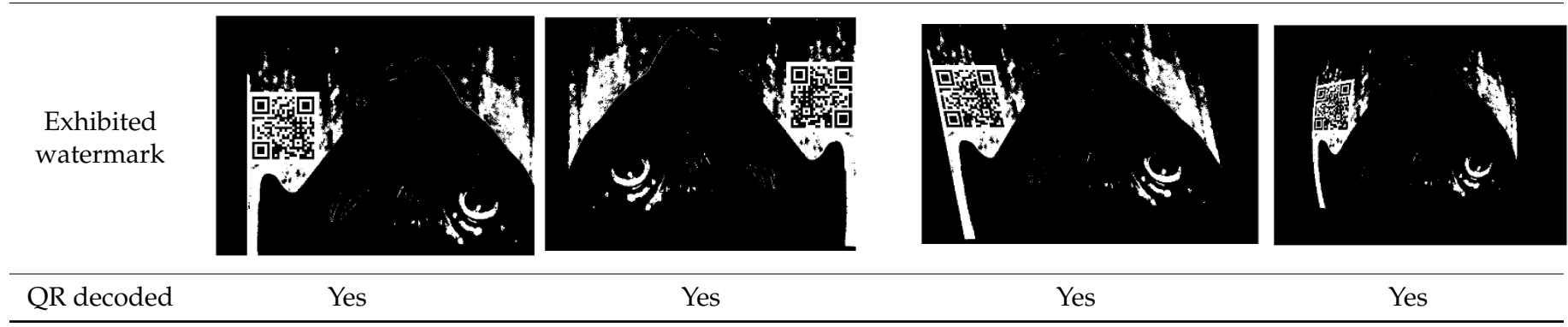

The high performance of the proposed algorithm against geometric attacks allows the recognition and information decoding of the watermark pattern $\mathrm{QR}$; however, robust affine transformation and aspect ratio attacks avoid the correctly contained information decoding from $\mathrm{QR}$ with error correction level $L$. To solve this drawback, a QR code with a higher error correction level could be used. 


\subsection{Robustness against Common Image Processing Attacks}

To evaluate the performance of the proposed algorithm against malicious or nonmalicious image processing attacks, an image is watermarked individually by the three proposed watermarks.

As example, Table 7 shows the QR watermarked image after being attacked by the common image processing attacks listed in Table 3. Table 8 shows the visual and numerical robustness results obtained from the images watermarked by the three mentioned watermarks. For the experimental evaluation, the quality of the exhibited watermarks is performed in terms of the NC and BER, and the watermarked images have been attacked by the maximum attack strength that the watermarks can resist; in this way, for watermarked images by $\mathrm{QR}$, a maximum attack strength allows the QR code information decoding to watermarked images by FI or UNAM, a maximum attack strength also allows recognizing the watermark; the specification to each attack is also provided. The obtained results show the robustness of the proposed algorithm against Filter, Noise contamination, Sharpening, Blurring, and Histogram Equalization; in all those cases, visual results of the exhibited watermarks can be recognized easily by the HVS. Numerical results in terms of NC and BER show diverse values nearest to 1 or 0 , respectively, which can be considered as the maximum or minimum degradation to recognize and/or decode the watermark pattern. Obtained results from the QR watermarked image show that the watermark can be exhibited with enough quality to be decoded after being attacked; however, its compression robustness is limited due to the $\mathrm{QR}$ watermark characteristics and its error correction level; in this way, the $\mathrm{QR}$ is only recognized after compression attacks.

Table 7. QR watermarked image after image processing attacks.

\begin{tabular}{|c|c|c|c|c|c|}
\hline \multirow{2}{*}{ Attack } & \multicolumn{3}{|c|}{ Filter } & \multicolumn{2}{|c|}{ Noise Contamination } \\
\hline & Average & Median & Gaussian & Impulsive & Gaussian \\
\hline \multicolumn{6}{|l|}{$\begin{array}{l}\text { Attacked } \\
\text { Image }\end{array}$} \\
\hline PSNR (dBs) & 30.10 & 31.44 & 38.49 & 9.92 & 45.05 \\
\hline \multirow{2}{*}{ Attack } & \multirow{2}{*}{ Sharpening } & \multirow{2}{*}{ Blurring } & \multirow{2}{*}{$\begin{array}{l}\text { Histogram } \\
\text { Equalization }\end{array}$} & \multicolumn{2}{|c|}{ Compression } \\
\hline & & & & JPEG & JPEG2000 \\
\hline \multicolumn{6}{|l|}{$\begin{array}{l}\text { Attacked } \\
\text { Image }\end{array}$} \\
\hline PSNR (dBs) & 39.31 & 29.95 & 8.02 & 36.72 & 41.07 \\
\hline
\end{tabular}


Table 8. Results of common image processing attacks.

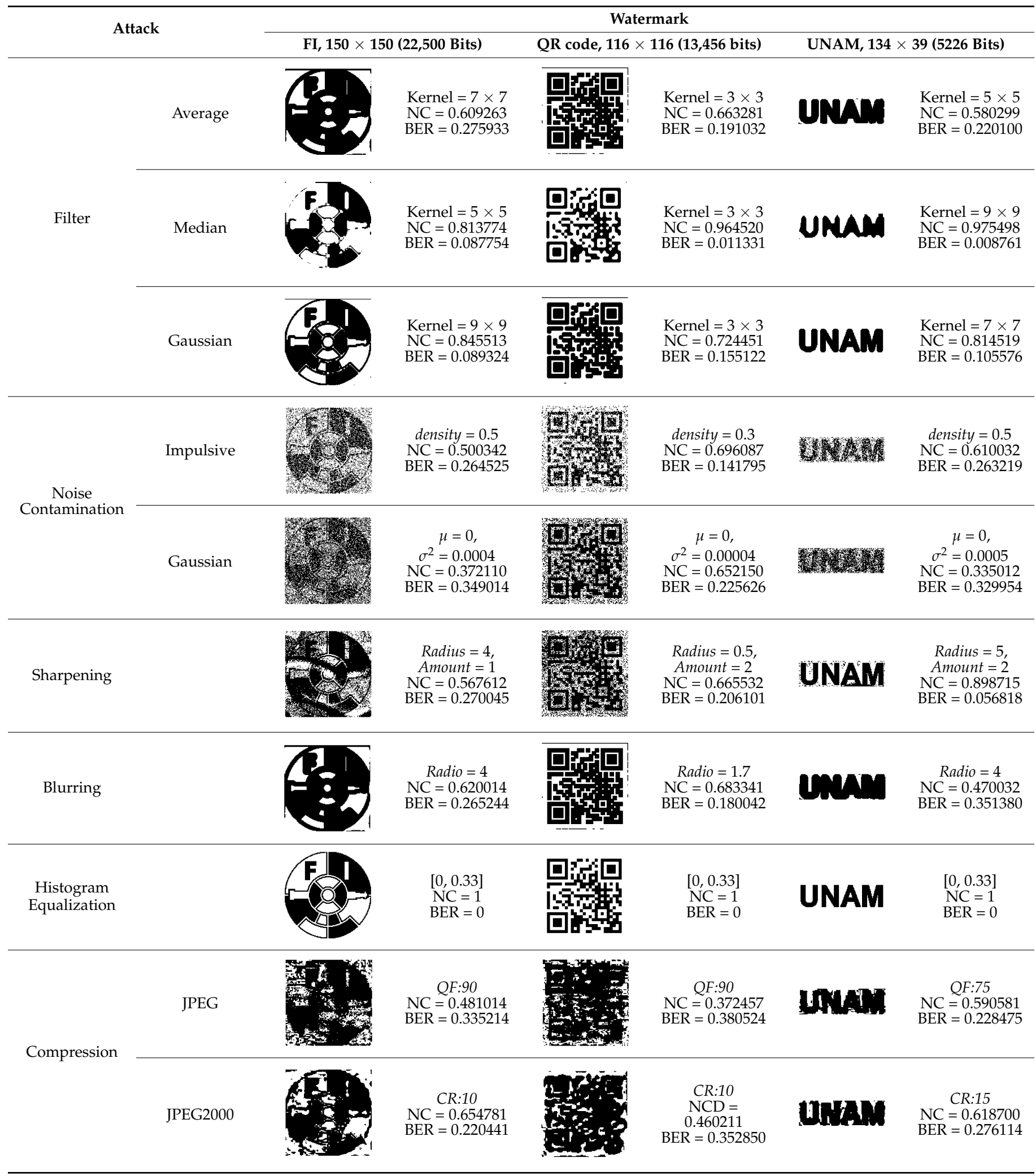

Similar to the results reported in [24], Table 8 shows that watermarks such as owner logotypes FI and UNAM are more robust against common image processing attacks than QR logotypes, as can be observed in the compression evaluation where a UNAM watermark is recognized after being compressed by JPEG and JPEG2000 with a quality factor QF:75 
and compression rate CR:15, respectively, while to recognize FI and QR watermarks, the compression attack must be decreased. In general, the proposed algorithm preserves a good imperceptibility and robustness against geometric and image processing attack.

\section{Comparison with Similar Algorithms}

In this section, the performance of the proposed algorithm is compared with the algorithms $[23,24]$ under the same characteristics such as hardwater capabilities, watermark, and image dataset.

To obtain a trustworthy result, 600 images randomly selected from the UCID dataset were watermarked by the algorithms [23,24], and the proposed watermark used in this evaluation is the QR code, because it is considered the most sensible watermark. Table 9 shows the evaluation results of quality and similarity of the watermarked images and the exhibited watermark patterns, respectively; this evaluation is obtained after treating the watermarked images by the image processing attacks listed in Table 3. As can be seen, the algorithm [23] shows better quality results due to its embedding strength being a fixed and empirical value of 2; in the case of the proposed algorithm, and in [24], the embedding strength is not a fixed value and could be selected by the corresponding strategy in the range of $[0,6]$. With respect to the similarity evaluation, [23] shows a poor performance due to the algorithm exhibiting the watermark in color. Finally, the proposed algorithm shows a better performance than [23] and one that is a little bit higher than [24].

Table 9. Robustness comparison against image processing attacks.

\begin{tabular}{|c|c|c|c|c|c|c|c|c|c|c|c|}
\hline \multirow{4}{*}{\multicolumn{2}{|c|}{ Attack }} & \multirow{4}{*}{ Specification } & \multicolumn{9}{|c|}{ Imperceptible Visible Watermarking } \\
\hline & & & \multicolumn{3}{|c|}{ Proposed } & \multicolumn{3}{|c|}{ [23] IVW } & \multicolumn{3}{|c|}{ [24] IIVW } \\
\hline & & & \multicolumn{3}{|c|}{ QR Code, $116 \times 116$} & \multicolumn{3}{|c|}{ QR Code, $116 \times 116$} & \multicolumn{3}{|c|}{ QR Code, $116 \times 116$} \\
\hline & & & $\begin{array}{l}\text { PSNR } \\
\text { (dB) }\end{array}$ & BER & NC & $\begin{array}{c}\text { PSNR } \\
\text { (dB) }\end{array}$ & BER & NC & $\begin{array}{c}\text { PSNR } \\
\text { (dB) }\end{array}$ & BER & NC \\
\hline \multirow{3}{*}{ Filter } & Average & $7 \times 7$ & 30.0994 & 0.2086 & 0.6546 & 31.1002 & 0.8995 & -0.7892 & 29.6237 & 0.1994 & 0.4205 \\
\hline & Median & $5 \times 5$ & 31.4374 & 0.0213 & 0.9550 & 31.4369 & 0.9785 & -0.9548 & 30.4635 & 0.3424 & 0.9288 \\
\hline & Gaussian & $9 \times 9$ & 38.4886 & 0.1642 & 0.7169 & 38.4905 & 0.9971 & -0.9939 & 38.7412 & 0.1922 & 0.7011 \\
\hline \multirow{2}{*}{$\begin{array}{l}\text { Noise con- } \\
\text { tamination }\end{array}$} & Impulsive & density $=0.5$ & 9.94921 & 0.1516 & 0.6860 & 9.9675 & 0.8506 & -0.6917 & 9.5478 & 0.1820 & 0.6104 \\
\hline & Gaussian & $\begin{array}{c}\mu=0 \\
\sigma^{2}=0.0004\end{array}$ & 45.0596 & 0.2267 & 0.6301 & 45.0465 & 0.8259 & -0.6299 & 45.3874 & 0.2163 & 0.5110 \\
\hline Sharpening & & $\begin{array}{l}\text { Radius }=4, \\
\text { Amount }=1\end{array}$ & 33.6065 & 0.2163 & 0.6442 & 33.6062 & 0.8041 & -0.5815 & 32.9437 & 0.2335 & 0.6157 \\
\hline Blurring & & Radio $=4$ & 29.9460 & 0.1958 & 0.6721 & 29.9468 & 0.9061 & -0.8031 & 28.3472 & 0.1742 & 0.6521 \\
\hline $\begin{array}{c}\text { Histogram } \\
\text { Equalization }\end{array}$ & & {$[0,0.33]$} & 8.0202 & 0 & 1 & 8.0200 & 0.4004 & -0.0907 & 8.0742 & 0 & 1 \\
\hline \multirow{2}{*}{ Compression } & JPEG & $Q F: 90$ & 36.7214 & 0.4067 & 0.3750 & 36.7312 & 0.6970 & -0.3472 & 35.9855 & 0.3210 & 0.3350 \\
\hline & JPEG2000 & CR:10 & 41.0719 & 0.3546 & 0.4566 & 41.0916 & 0.7330 & -0.4242 & 40.4582 & 0.3308 & 0.4544 \\
\hline
\end{tabular}

Figure 11a,b shows a BER comparison of the exhibited QR watermark after compression by JPEG and JPEG 2000, respectively; in both images L, M, Q, and H correspond to the QR error correction level. 


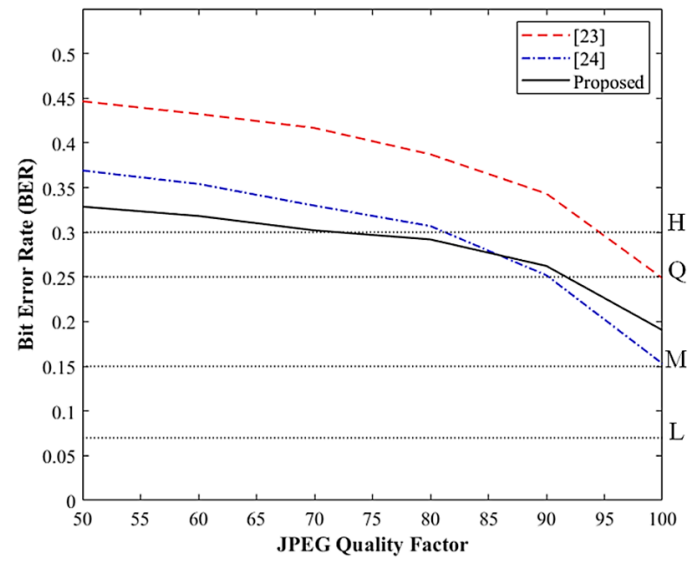

(a)

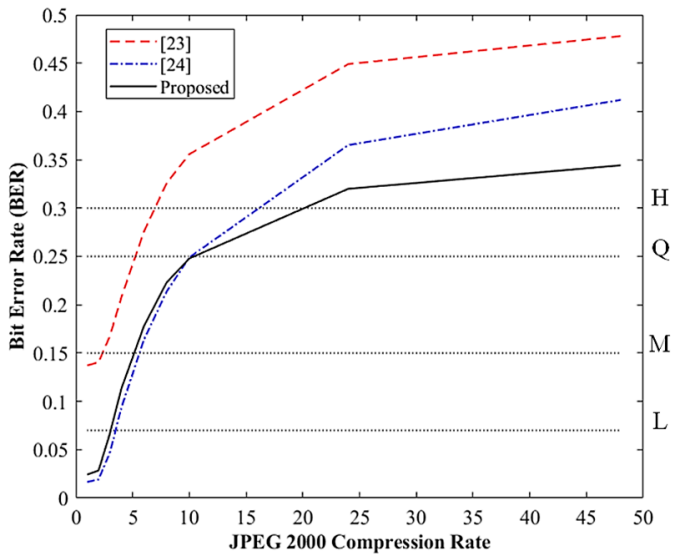

(b)

Figure 11. Performance comparison in terms of compression. (a) BER vs. JPEG, (b) BER vs. JPEG2000.

Table 10 compares the image quality degradation and watermark imperceptibility of the proposed algorithm against those of [23] and [24]. In this evaluation, we used the 600 images that have been watermarked by the QR code with the maximum embedding strength. As can be seen, the obtained results for [23] are similar to those proposed; however, as explained previously, the embedding strength of [23] corresponds to the empirical value of 2, while the embedding strength of [24] and the proposed algorithm corresponds to $\Delta=5$.

Table 10. Image quality and watermark imperceptibility comparison.

\begin{tabular}{cccc}
\hline Algorithm & Image Quality & \multicolumn{2}{c}{ Image Imperceptibility } \\
\hline & PSNR & SSIM & NCD \\
{$[23]$} & 50.4186 & 0.9978 & 0.0040 \\
{$[24]$} & 48.5215 & 0.9951 & 0.0074 \\
Proposed & 51.2145 & 0.9963 & 0.0060 \\
\hline
\end{tabular}

\section{Discussion and Analysis}

To improve the previously reported algorithm [23], the proposed algorithm takes advantage of the histogram distortion induced by the embedding strategy. The obtained results show that the proposed algorithm has better performance than the most sophisticated and recent algorithm [24]. These results are obtained considering that the embedding region is selected as the largest homogeneous region satisfying the minimum variance; however, in a real-world scenario, the captured scenes can integrate many homogeneous regions that make it possible to embed multiple watermarks. Figure 12a shows an image that 
once analyzed chooses three non-overlapping regions to embed the proposed watermarks; Figure $12 b-d$ show the corresponding exhibited watermarks.

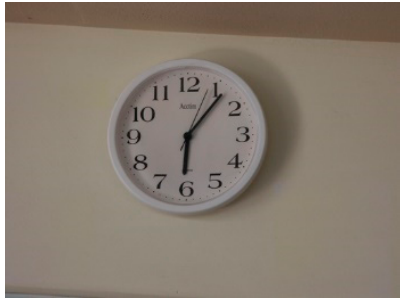

(a)

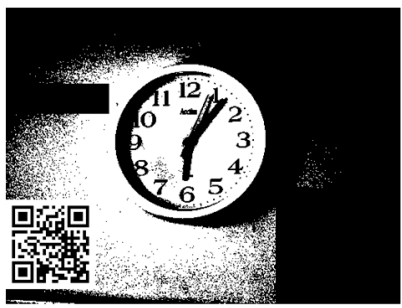

(c)

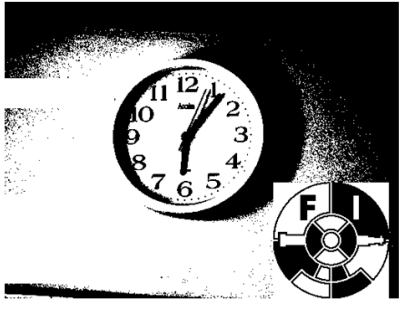

(b)

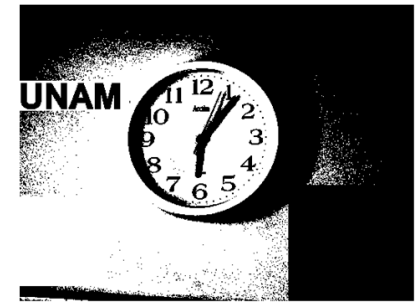

(d)

Figure 12. Multi-Watermarked image, (a) Watermarked image PSNR $=48.63 \mathrm{~dB}, \mathrm{SSIM}=0.9964$, NDC $=0.0055$, (b) Exhibited FI watermark, (c) Exhibited QR watermark, (d) Exhibited UNAM watermark.

The relation between the embedding and the exhibition strategy of [23,24], and the proposed algorithm is analyzed by the histogram distortion of the embedding region; this relation makes it possible to share the exhibition strategies among them, mainly the histogram modulation-based strategy. Figure 13a is the same multi-watermarked image of Figure 12a, and Figure 13b-d show the results to share among them regarding histogram modulation as the exhibition strategy. These results are possible due to all the algorithms inducing the same histogram distortion in the appropriate embedding region; however, this strategy is not feasible to exhibit QR codes.

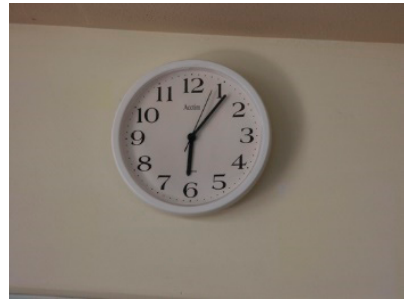

(a)

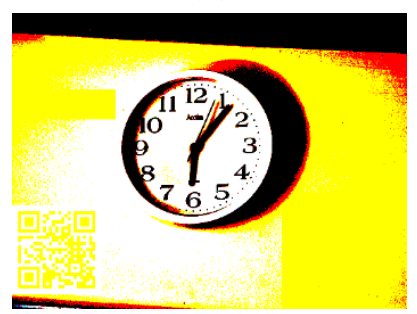

(c)

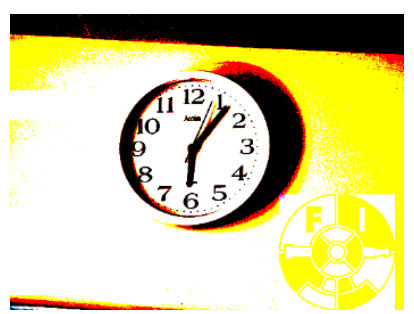

(b)

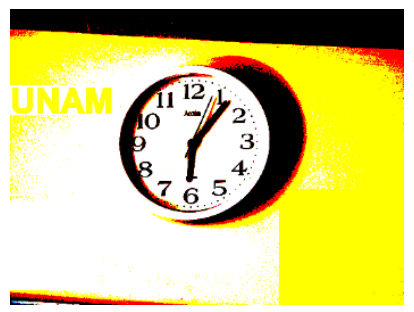

(d)

Figure 13. Histogram modulation-based exhibition strategy applied to the proposed algorithm, (a) Multi-Watermarked image, (b) Exhibited FI watermark, (c) Exhibited QR watermark, (d) Exhibited UNAM watermark. 
In [24], the reported algorithm is applied on streaming video and images services, where end-users can exhibit the watermark through clicking a media-player button, which executes the corresponding exhibition strategy. To exhibit the watermark, the exhibition strategy shares a crucial parameters set that was embedded previously by the invisible watermarking algorithm QIM-DM [28], the incorporation of this algorithm increasing the computational cost and decreasing the image quality. In this context and taking into account that the most popular on-demand and streaming services, such as Netflix [44], DisneyPlus [45], and PrimeVideo [46], require a dedicated platform to play their audiovisual content, the crucial parameters to exhibit the watermark can be transmitted by the platform and stored into the memory of the electronic display device. When the end-users want to exhibit the watermark, the proposed algorithm takes advantage of robust image processing capabilities; with this strategy, an additional complex computational algorithm is not required to exhibit the watermark. If the proposed algorithm considers the same crucial parameters to exhibit every watermark, the required memory and the computational cost are low.

\section{Conclusions}

In this paper, a copyright protection and information delivery algorithm is presented; the proposed one introduces a new improved version of the pioneer imperceptible-visible watermarking algorithm [23] that takes advantage of the histogram distortion induced by the embedding strategy to introduce a new embedding strategy, and the processing capabilities incorporated into the electronic display devices to propose a new histogram binarization function-based exhibition strategy. The experimental results show that the proposed algorithm improves several aspects of the most sophisticated and recent IVW algorithm [24]. In general, the algorithm can embed one or multiple watermarks, guaranteeing high watermarked image quality in the range of $37 \mathrm{~dB} \leq P S N R \geq 53 \mathrm{~dB}$, and the watermark imperceptibility is reported with an SSIM $\geq 0.93$ and NCD $\leq 0.05$, preserving the high visual quality of the exhibited watermarks, mainly QR codes. The low complexity of the proposed algorithm makes it a feasible option to be applied to copyright protection and information delivery related to video or image visual content.

Author Contributions: Conceptualization, O.U.J.-S. and F.J.G.-U.; methodology, F.J.G.-U., M.C.-H. and L.H.-G.; software, O.U.J.-S.; validation, O.U.J.-S., F.J.G.-U. and M.C.-H.; formal analysis, O.U.J.S., F.J.G.-U. and M.C.-H.; investigation, O.U.J.-S., F.J.G.-U. and M.C.-H.; resources, M.C.-H. and L.H.-G.; data curation, O.U.J.-S. and J.R.-H.; writing, original draft preparation, O.U.J.-S. and J.R.H.; writing, review and editing, O.U.J.-S. and J.R.-H.; visualization, F.J.G.-U., M.C.-H. and L.H.-G.; supervision, F.J.G.-U., M.C.-H. and L.H.-G.; project administration, F.J.G.-U., M.C.-H. and L.H.-G.; funding acquisition, F.J.G.-U. and L.H.-G. All authors have read and agreed to the published version of the manuscript.

Funding: This research was funded by the Universidad Nacional Autónoma de México (UNAM) under the DGAPA Postdoctoral Scholarship Program and the PAPIIT-IT101119 Research Project, and by the Instituto Politécnico Nacional (IPN).

Institutional Review Board Statement: Not applicable.

Data Availability Statement: Publicly available datasets were analyzed in this study. These data can be found here: https:/ / www.dropbox.com/s/3alzvk6z8yumeik/Aditional\%20IVW\%20Re-598sults. rar? $\mathrm{dl}=0$ (accessed on 20 September 2021).

Acknowledgments: The authors thank the Universidad Nacional Autónoma de México (UNAM) under the DGAPA Postdoctoral Scholarship Program, the PAPIIT-IT101119 Research Project, and the Instituto Politécnico Nacional (IPN) by the support provided during the realization of this research. The authors of the article appreciate the valuable suggestions of the referees, and the editor who contributed to improve the paper.

Conflicts of Interest: The authors declare no conflict of interest. 


\section{References}

1. Cox, I.J.; Kilian, J.; Leighton, F.T.; Shamoon, T. Secure spread spectrum watermarking for multimedia. IEEE Trans. Image Process. 1997, 6, 1673-1687. [CrossRef]

2. Cox, I.J.; Miller, M.; Bloom, J.; Fridrich, J.; Kalker, T. Digital Watermarking and Steganography, 2nd ed.; Morgan Kaufmann: San Francisco, CA, USA, 2007; pp. 335-374.

3. Barni, M.; Bartolini, F. Watermarking Systems Engineering: Enabling Digital Assets Security and Other Applications (Signal Processing and Communications), 1st ed.; CRC Press: Boca Raton, FI, USA, 2004; pp. 23-43.

4. Lu, C.S. Steganography and Digital Watermarking Techniques for Protection of Intellectual Property; Idea Group Publishing: Hershey, PA, USA, 2005; pp. 173-206.

5. Singh, P.; Chadha, R.S. A survey of digital watermarking techniques, applications and attacks. Int. J. Eng. Innov. Technol. 2013, 2, 165-175.

6. Singh, A.K. Some New Techniques of Improved Wavelet Domain Watermarking for Medical Images. Ph.D. Thesis, National Institute of Technology, Kurukshetra, Haryana, India, May 2015.

7. Su, Q.; Liu, D.; Yuan, Z.; Wang, G.; Zhang, X.; Chen, B.; Yao, T. New rapid and robust color image watermarking technique in spatial domain. IEEE Access 2019, 7, 30398-30409. [CrossRef]

8. Langelaar, G.; Setyawan, I.; Lagendijk, R. Watermarking digital image and video data. A state-of-the-art overview. IEEE Signal Process. Mag. 2000, 17, 20-46. [CrossRef]

9. Li, J.; Zhang, H.; Wan, W.; Sun, J. Two-class 3D-CNN classifiers combination for video copy detection. Multimed. Tools Appl. 2020, 79, 4749-4761. [CrossRef]

10. Iwendi, C.; Jalil, Z.; Javed, A.R.; Reddy, G.T.; Kaluri, R.; Srivastava, G.; Jo, O. Key Split Watermark: Zero watermarking algorithm for software protection against cyber-attacks. IEEE Access 2020, 8, 72650-72660. [CrossRef]

11. Zigomitros, A.; Papageorgiou, A.; Patsakis, C. Social network content management through watermarking. In Proceedings of the IEEE 11th International Conference on Trust, Security and Privacy in Computing and Communications, Liverpool, UK, 25-27 June 2012; pp. 1381-1386.

12. Bertini, F.; Sharma, R.; Iannì, A.; Montesi, C. Smartphone verification and user profiles linking across social networks by camera fingerprinting. In Proceedings of the 7th International Conference on Digital Forensics and Cyber Crime, Seoul, Korea, 6-8 October 2015; pp. 176-186.

13. Park, Y.; Kim, J.; Kim, M.; Lee, W.; Lee, S. Programmable multimedia platform based on reconfigurable processor for 8K UHD TV. IEEE Trans. Consum. Electron. 2015, 61, 516-523. [CrossRef]

14. Wang, H.; Zhang, X.; Wang, T.; Li, W.; Chen, Q.; Ren, P.; Wu, X.; Sun, H. A 4K × 2K@60fps Multifunctional Video Display Processor for High Perceptual Image Quality. IEEE Trans. Circuits Syst. I Regul. Pap. 2020, 67, 451-463. [CrossRef]

15. Caviedes, J.E. The Evolution of Video Processing Technology and Its Main Drivers. Proc. IEEE 2012, 100, 872-877. [CrossRef]

16. Xu, C.; Peng, Z.; Hu, X.; Zhang, W.; Chen, L.; An, F. FPGA-Based Low-Visibility Enhancement Accelerator for Video Sequence by Adaptive Histogram Equalization With Dynamic Clip-Threshold. IEEE Trans. Circuits Syst. I Regul. Pap. 2020, 67, $3954-3964$. [CrossRef]

17. Li, Z.; Wang, J.; Sylvester, D.; Blaauw, D.; Kim, H.S. A $1920 \times 1080$ 25-Frames/s 2.4-TOPS/W Low-Power 6-D Vision Processor for Unified Optical Flow and Stereo Depth With Semi-Global Matching. IEEE J. Solid-State Circuits 2019, 54, 1048-1058. [CrossRef]

18. Chuang, S.; Huang, C.; Wu, J. Unseen Visible Watermarking. In Proceedings of the 2007 IEEE International Conference on Image Processing, San Antonio, TX, USA, 16 September-19 October 2007.

19. Huang, C.; Chuang, S.; Huang, Y.; Wu, J. Unseen Visible Watermarking: A Novel Methodology for Auxiliary Information Delivery via Visual Contents. IEEE Trans. Inf. Forensics Secur. 2009, 4, 193-206. [CrossRef]

20. Juarez-Sandoval, O.; Fragoso-Navarro, E.; Cedillo-Hernandez, M.; Nakano, M.; Perez-Meana, H.; Cedillo-Hernandez, A. Improved unseen-visible watermarking for copyright protection of digital image. In Proceedings of the 5th International Workshop on Biometrics and Forensics (IWBF), Coventry, UK, 4-5 April 2017; pp. 1-5.

21. Juarez-Sandoval, O.; Cedillo-Hernandez, M.; Nakano, M.; Cedillo-Hernandez, A.; Perez-Meana, H. Digital image ownership authentication via camouflaged unseen-visible watermarking. Multimed. Tools Appl. 2018, 77, 26601-26634. [CrossRef]

22. Hsieh, W.; Lin, P. Imperceptible Visible Watermarking Scheme Using Color Distribution Modulation. In Proceedings of the 9th International Conference on Ubiquitous Intelligence and Computing and International Conference on Autonomic and Trusted Computing, Fukuoka, Japan, 4-7 September 2012; pp. 1002-1005.

23. Lin, P.Y. Imperceptible Visible Watermarking Based on Postcamera Histogram Operation. J. Syst. Softw. 2014, 95, 194-208. [CrossRef]

24. Juarez-Sandoval, O.; Fragoso-Navarro, E.; Cedillo-Hernandez, M.; Cedillo-Hernandez, A.; Nakano, M.; Perez-Meana, H. Improved imperceptible visible watermarking algorithm for auxiliary information delivery. IET Biom. 2018, 7, 305-313. [CrossRef]

25. Juarez-Sandoval, O.U.; Garcia-Ugalde, F.; Cedillo-Hernandez, M.; Ramirez-Hernandez, J. Imperceptible Visible Watermarking with Watermark Readability Improved. In Proceedings of the IEEE International Autumn Meeting on Power, Electronics and Computing (ROPEC), Guerrero, México, 4-6 November 2020; pp. 1-6.

26. Projected Image System Contrast Ratio, ANSI/INFOCOMM 3M-2011. Available online: https://www.avixa.org/standards/ projected-image-system-contrast-ratio (accessed on 12 April 2021). 
27. Gamma Correction Function. Available online: https://www.mathworks.com/help/images/ref/imadjust.html?s_tid=srchtitle (accessed on 1 July 2021).

28. Chen, B.; Wornell, G.W. Quantization index modulation: A class of provably good methods for digital watermarking and information embedding. IEEE Trans. Inf. Theory 2001, 47, 1423-1443. [CrossRef]

29. Yu, P.; Shang, Y.; Li, C. A new visible watermarking technique applied to CMOS image sensor. In Proceedings of the MIPPR 2013: Multispectral Image Acquisition, Processing, and Analysis, Wuhan, China, 26-27 October 2013; p. 891719.

30. Bae, S.; Kim, M. A Novel DCT-Based JND Model for Luminance Adaptation Effect in DCT Frequency. IEEE Signal Process. Lett. 2013, 20, 893-896.

31. Fragoso-Navarro, E.; Cedillo-Hernandez, M.; Nakano-Miyatake, M.; Cedillo-Hernandez, A.; Pérez-Meana, H.M. Visible Watermarking Assessment Metrics Based on Just Noticeable Distortion. IEEE Access 2018, 6, 75767-75788. [CrossRef]

32. Yang, X.K.; Lin, W.S.; Lu, Z.K.; Ong, E.P.; Yao, S.S. Just noticeable distortion model and its applications in video coding. Signal Process. Image Commun. 2005, 20, 662-680. [CrossRef]

33. Jung, S.; Ha, L.T.; Ko, S. A New Histogram Modification Based Reversible Data Hiding Algorithm Considering the Human Visual System. IEEE Signal Process. Lett. 2011, 18, 95-98. [CrossRef]

34. Liu, H.; Zhang, Y.; Zhang, H.; Fan, C.; Kwong, S.; Jay-Kuo, C.-C.; Fan, X. Deep Learning-Based Picture-Wise Just Noticeable Distortion Prediction Model for Image Compression. IEEE Trans. Image Process. 2020, 29, 641-656. [CrossRef] [PubMed]

35. Petitcolas, F.A.P.; Anderson, R.J.; Kuhn, M.G. Attacks on copyright marking systems. In Proceedings of the International Workshop on Information Hiding, Berlin, Germany, 14-17 April 1998; pp. 218-238.

36. Schaefer, G.; Stich, M. UCID: An uncompressed color image database. In Proceedings of the Storage and Retrieval Methods and Applications for Multimedia, San Jose, CA, USA, 18-22 January 2004; pp. 472-480.

37. Quick Response Code. Available online: https://www.qrcode.com/ (accessed on 5 July 2021).

38. Wang, Z.; Bovik, A.C.; Sheikh, H.R.; Simoncelli, E.P. Image quality assessment: From error visibility to structural similarity. IEEE Trans. Image Process. 2004, 13, 600-612. [CrossRef]

39. Chang, H.; Chen, H.H. Stochastic Color Interpolation for Digital Cameras. IEEE Trans. Circuits Syst. Video Technol. 2007, 17, 964-973. [CrossRef]

40. Supplemental Results. Available online: https://www.dropbox.com/s/3alzvk6z8yumeik/Aditional\%20IVW\%20Results.rar?dl= 0 (accessed on 20 September 2021).

41. Guo, Y.; Li, B.Z.; Goel, N. Optimized blind image watermarking method based on firefly algorithm in DWT-QR transform domain. IET Image Process. 2017, 11, 406-415. [CrossRef]

42. Snapseed. Available online: https:/ / snapseed.uptodown.com (accessed on 1 July 2021).

43. Adobe Photoshop Express and Adobe Lightroom. Available online: https://www.adobe.com/ (accessed on 24 July 2021).

44. Netflix. Available online: https:/ / www.netflix.com/ (accessed on 11 July 2021).

45. Disney Plus. Available online: https:/ / www.disneyplus.com/. (accessed on 11 July 2021).

46. Prime Video. Available online: https://www.primevideo.com/ (accessed on 11 July 2021). 